\title{
Erratum to: A Study of Heating Duration and Scanning Path in Focused Ultrasound Surgery
}

Dehui Li • Guofeng Shen • Hui Luo • Jingfeng Bai • Yazhu Chen

Published online: 7 January 2012

(C) Springer Science+Business Media, LLC 2012

Erratum to: J Med Syst (2011) 35:779-786

DOI 10.1007/s10916-010-9463-6

The original version of this article unfortunately contained a mistake. One author name is incorrect, Jinfeng Bai should be Jingfeng Bai.

The online version of the original article can be found at http://dx.doi. org/10.1007/s10916-010-9463-6.

D. Li $\cdot$ G. Shen $(\varangle) \cdot$ H. Luo $\cdot$ J. Bai $\cdot$ Y. Chen

Biomedical Instrument Institute, Shanghai Jiao Tong University,

Shanghai 200030, China

e-mail: shenguofeng@sjtu.edu.cn

D. Li $\cdot$ G. Shen $\cdot$ H. Luo $\cdot$ J. Bai $\cdot$ Y. Chen

Med-X Research Institute, Shanghai Jiao Tong University,

Shanghai 200030, China 